\title{
Union of Scaphoid Waist Fractures in Adults Despite No or Minimal Immobilization-a Report of Five Cases
}

\author{
Lars Adolfsson $^{1} \cdot$ Hanna Björnsson Hallgren ${ }^{1} \cdot$ Yelverton Tegner ${ }^{2}$
}

Accepted: 19 March 2020 / Published online: 27 March 2020

(C) The Author(s) 2020

\begin{abstract}
Traditionally scaphoid waist fractures have been treated with a long period, 10-12 weeks, of immobilization in a plaster cast until radiological union. The long period of immobilization sometimes infers great discomfort for the patients. To avoid this, surgical treatment with screw fixation has for the last decade been advocated as an option also for undisplaced fractures. In this report, we present five cases with undisplaced scaphoid waist fractures that have healed both radiologically and clinically without any immobilization at all or after a very short period of protection in a cast. These observations challenge the current treatment guidelines.
\end{abstract}

Keywords Scaphoid fracture $\cdot$ Sports $\cdot$ Conservative treatment $\cdot$ Union

\section{Introduction}

Scaphoid waist fractures without significant displacement are usually treated non-operatively by immobilization in a cast. Traditionally, most have recommended cast immobilization until bridging bone trabeculae is seen which often has rendered a plaster cast for 10-12 weeks. Time to radiographic union may however vary, and more recently, shorter immobilization for approximately 6 weeks has been found sufficient for undisplaced fractures, and using this management union has been reported in approximately $90 \%$ [3]. Acute internal fixation with screws has been found to produce results equal to traditional conservative treatment $[1,5]$ without the need for long periods of immobilization in plaster cast, and there seems to have been an increasing trend in favour of acute internal fixation $[4,7]$.

Level of evidence: IV case series

This article is part of the Topical Collection on Surgery

Hanna Björnsson Hallgren

hanna.bjornsson.hallgren@regionostergotland.se

1 Department of Orthopaedics; Institution of Surgery and Experimental medicine, Linköping University, 58185 Linköping, Sweden

2 Division of Medical Sciences, Department of Health Sciences, Luleå University of Technology, 97187 Luleå, Sweden
There may be several patient-related factors such as age, smoking, but also fracture-specific factors for instance vascularity of the fracture fragments, instability of the fracture and fracture pattern that can have an impact on healing potential. This means that the time to union and the tendency for healing disturbances may vary depending on inherent fracture characteristics.

The following report describes five patients in whom union of an undisplaced scaphoid waist fracture took place without or after a very short time of immobilization. All patients were healthy, non-smokers, and four out of five were elite athletes. These observations underline the importance of recognition of all aspects influencing fracture healing and challenge many of the prevailing treatment guidelines. All patients have consented publication of the included information and radiographs.

\section{Case Report 1}

A 24-year-old, professional, ice hockey player sustained a dorsiflexion trauma to his non-dominant left wrist during a game when he was checked into the board. Due to persisting radial-sided wrist pain, he sought medical advice 31 days later, after having fully participated in training and games in the meantime. Standard $\mathrm{x}$-rays were taken and revealed a nondisplaced scaphoid waist fracture (Fig. 1a). He was recommended not to take part in games but was allowed training with the use of an orthosis immobilizing the wrist and thumb base. Two weeks later, a new x-ray (Fig. 1b) demonstrated 

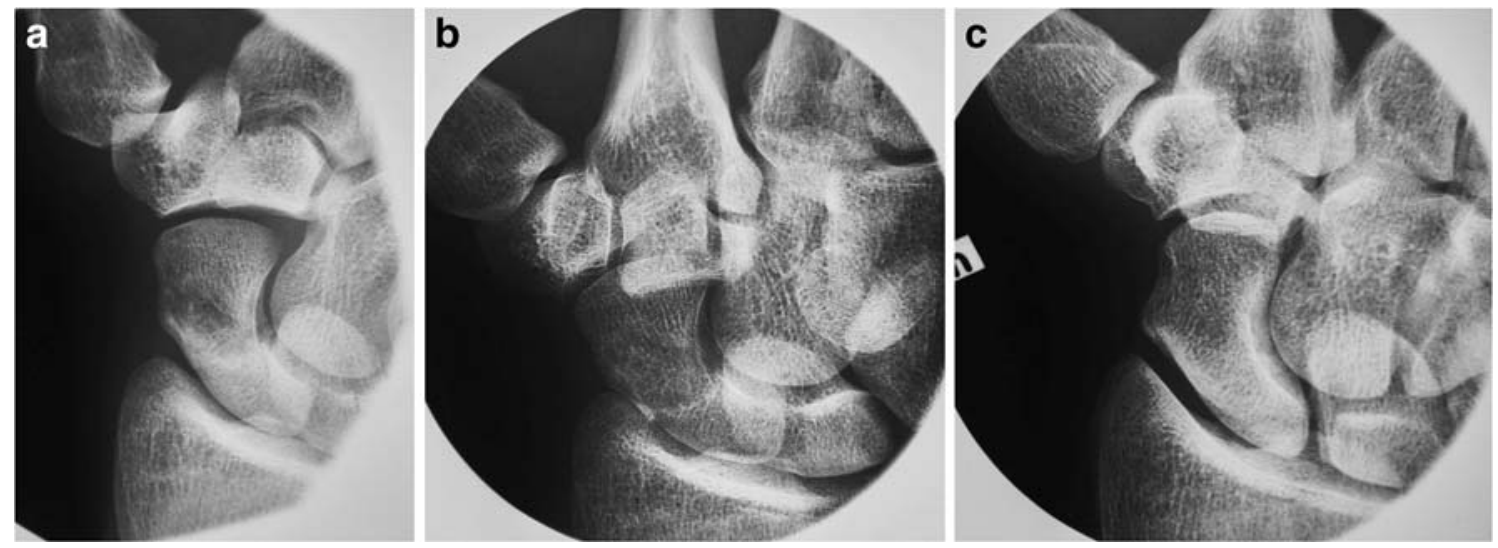

Fig. 1 a Radiograph taken 31 days after a dorsiflexion trauma demonstrating a non-displaced scaphoid waist fracture, b new images 2 weeks later showing signs of slight compression, $\mathbf{c}$ union confirmed at a control 7 years later

slight compression of the fracture and beginning callus formation according to the radiologist. The symptoms had markedly improved, and the patient resumed full activity resuming ice hockey training and matches without restrictions. At 10 weeks, the fracture was assessed as united, and at a final control after 7 years, an uneventful union of the fracture was seen (Fig. 1c).

\section{Case Report 2}

Another, 25-year-old, male, professional, ice hockey player injured his non-dominant left wrist during a game when he collided with a player in the opposing team. He had an x-ray the following day demonstrating a non-displaced, transverse fracture in the proximal part of the middle third of the scaphoid (Fig. 2a). He was immobilized in a cast, and the fracture remained in the same position according to a control x-ray without the plaster 19 days after the injury. Following this examination, he wanted the cast to be removed, and it was replaced by a short orthotic brace. Despite recommendations of a slower rehab, the patient resumed full training with his team within 5 weeks after the initial injury. Consolidation of the fracture was seen at a follow-up x-ray 4 months after the injury (Fig. 2b). He continued his professional career several years after this injury without any wrist complaints.

\section{Case Report 3}

A 16-year-old, male, ice hockey player sustained a dorsiflexion injury to his right wrist during a game. He complained over radial-sided wrist pain and was examined with conventional $\mathrm{x}$ rays the same day. The scaphoid projections displayed a transverse, undisplaced, waist fracture (Fig. 3a), and he was immobilized in a below elbow cast. After 21 days, he had a control x-ray without the plaster, and since the radiologist described signs of callus formation and the patient had no pain or tenderness in the anatomical snuff box, the cast was removed (Fig. 3b). He went back to full activity including ice hockey training and matches 6 weeks after the initial injury and remained free of symptoms for the rest of a long career as a professional player. A control radiograph was taken 3 years after the initial injury and demonstrated complete union (Fig. 3c).

\section{Case Report 4}

A 34-year-old male carpenter injured his right wrist when falling with his motorbike. Initial $\mathrm{X}$-rays were suggestive of an undisplaced fracture but regarded as inconclusive, and a CT scan was performed, also indicating an undisplaced crack at the scaphoid waist (Fig. 4a and b). He was further examined with a MRI the following day demonstrating a transverse, undisplaced fracture with a surrounding bone oedema (Fig. 4c). The patient was instructed to avoid heavy load on the wrist during 6 weeks but was not immobilized and was allowed unrestricted use of the hand in everyday activities. A control x-ray after 10 weeks demonstrated union, and a MRI control at 5 months showed healing of the fracture (Fig. 4d). He remained completely asymptomatic at the latest follow-up 5 years after the injury.

\section{Case Report 5}

A professional, 23-year-old, female, basketball player fell during the first game of an international tournament. She complained over severe pain in the radial side of her right wrist, and after the game, a MRI scan was performed the same day at a nearby hospital, which demonstrated a transverse line across the scaphoid waist. The finding was found consistent with an undisplaced fracture (Fig. 5a). She was recommended immobilization in a cast, but since she wanted to continue the tournament, she went on to play another four games during the following 10 days, 
Fig. 2 a Radiographs of the left wrist the day after a trauma demonstrating a scaphoid waist fracture, $\mathbf{b}$ the wrist was immobilized 19 days, and union of the fracture was confirmed with radiographs after 4 months a

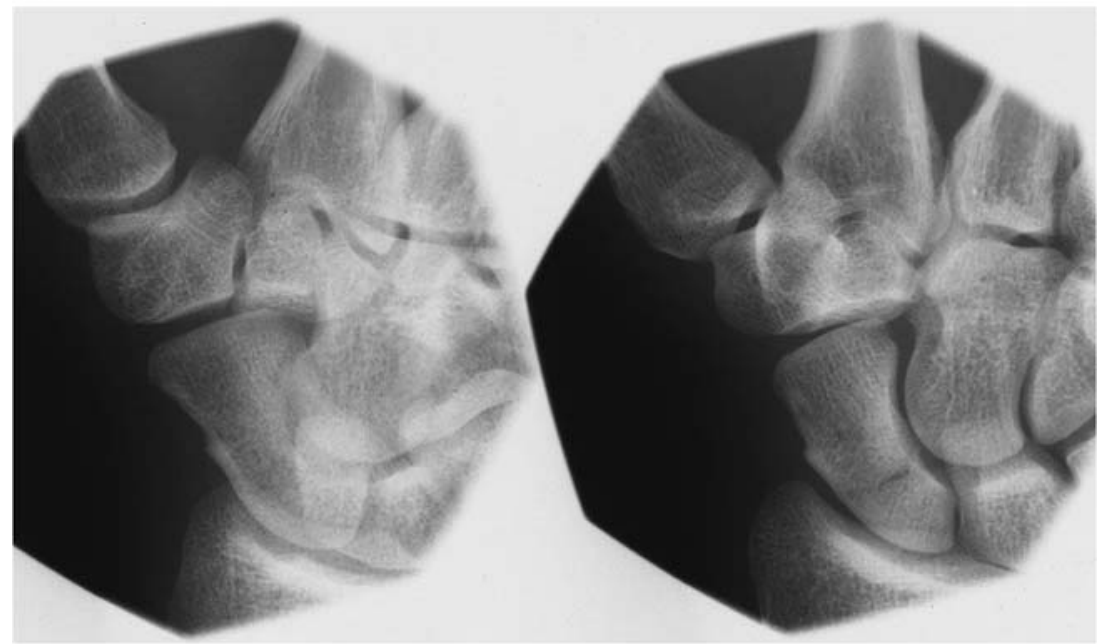

b

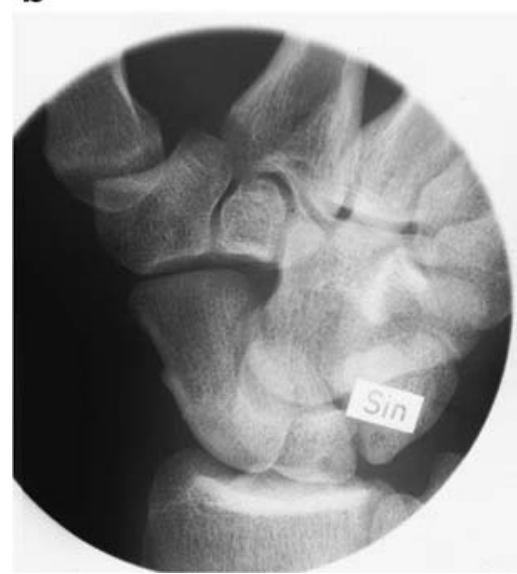

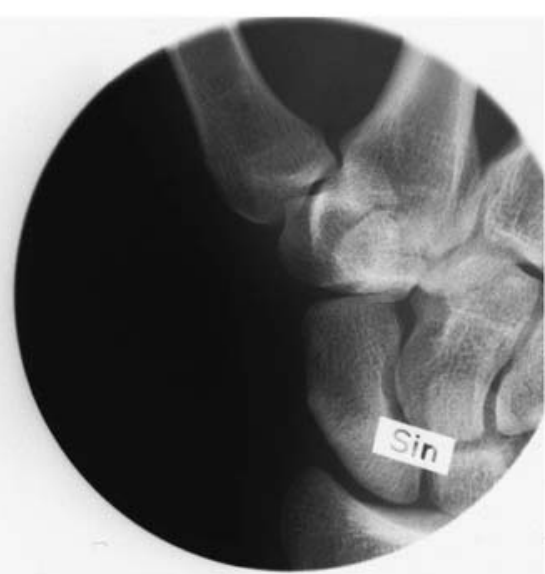

protecting the injured wrist with tape during the matches. After completing the tournament, on her returning home, another examination with a CT scan was undertaken showing an undisplaced fracture. She was then treated with a short splint immobilizing the wrist and thumb base. Due to a severe allergic skin reaction, she however had the
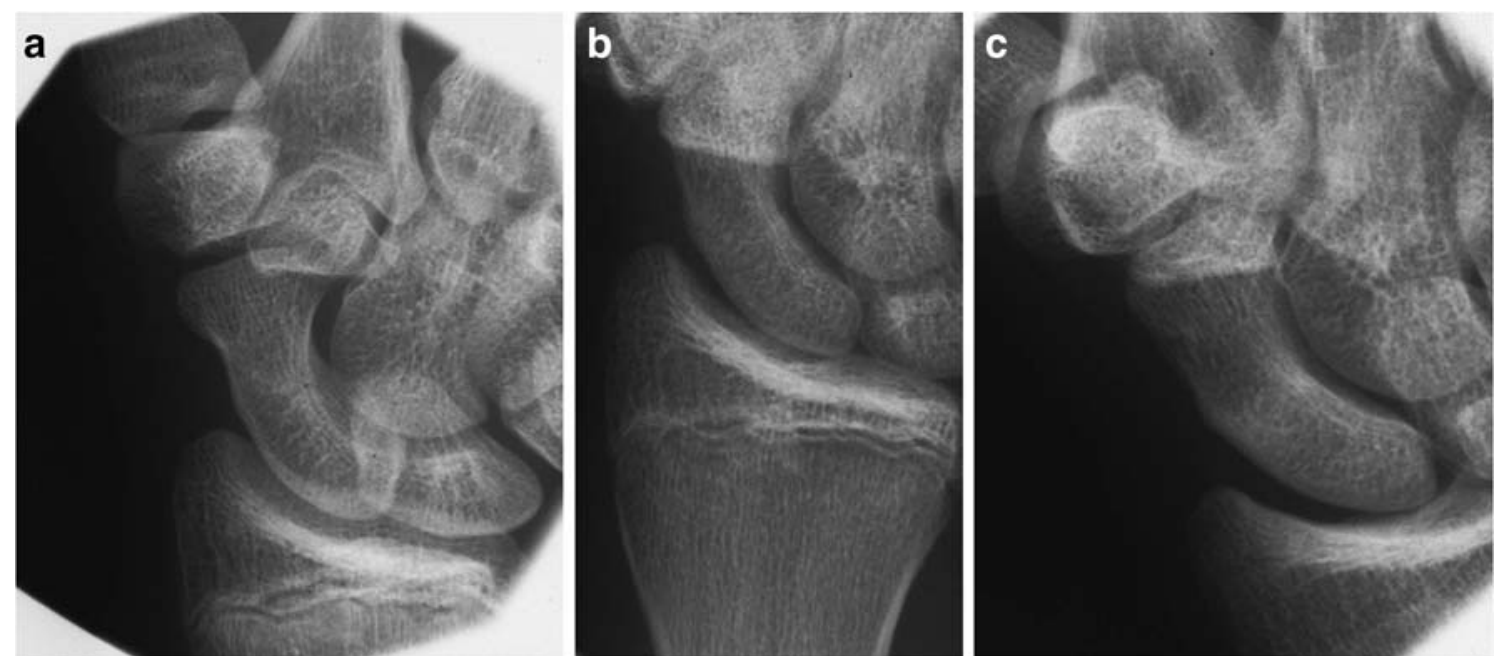

Fig. 3 a. The same day as the trauma, scaphoid projections displayed a transverse, undisplaced, waist fracture, b. New images without cast 21 days after the trauma, c. A control radiograph three years later showing complete union 
Fig. 4 a Following a fall a 34year-old male complained over radial-sided wrist pain.

Conventional $\mathrm{x}$-rays suggestive of a scaphoid waist fracture, $\mathbf{b}$ a CT scan the same day as the injury also indicated a fracture but was regarded as inconclusive, c. a MRI the following day was assessed as confirmative of the diagnosis, $\mathbf{d}$ MRI control

4 months later shows union in progress
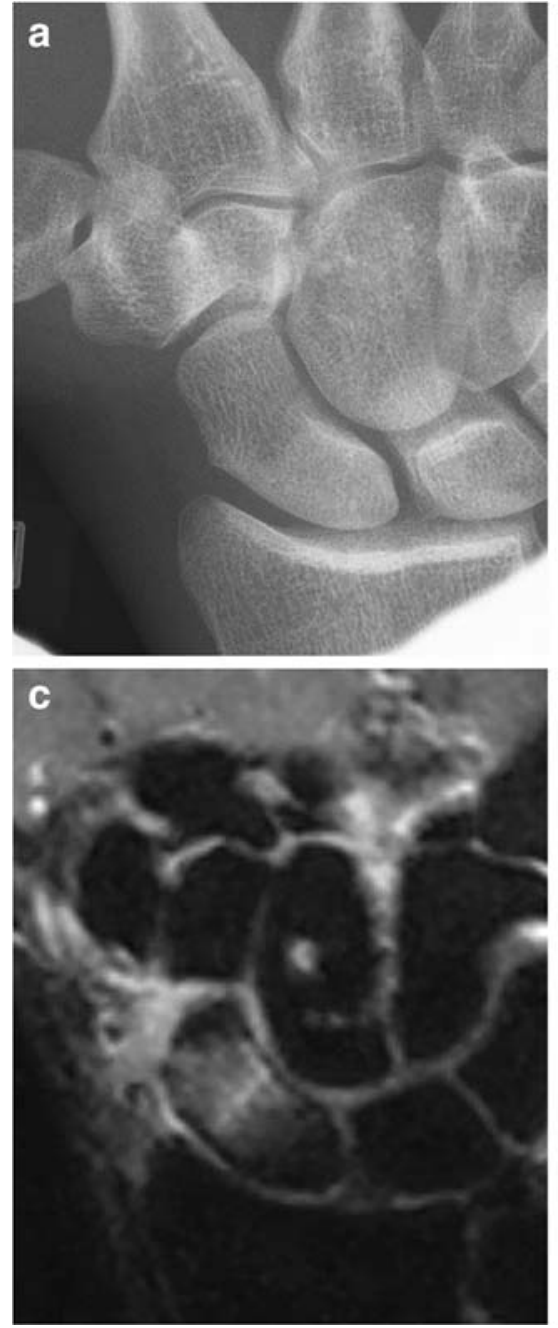
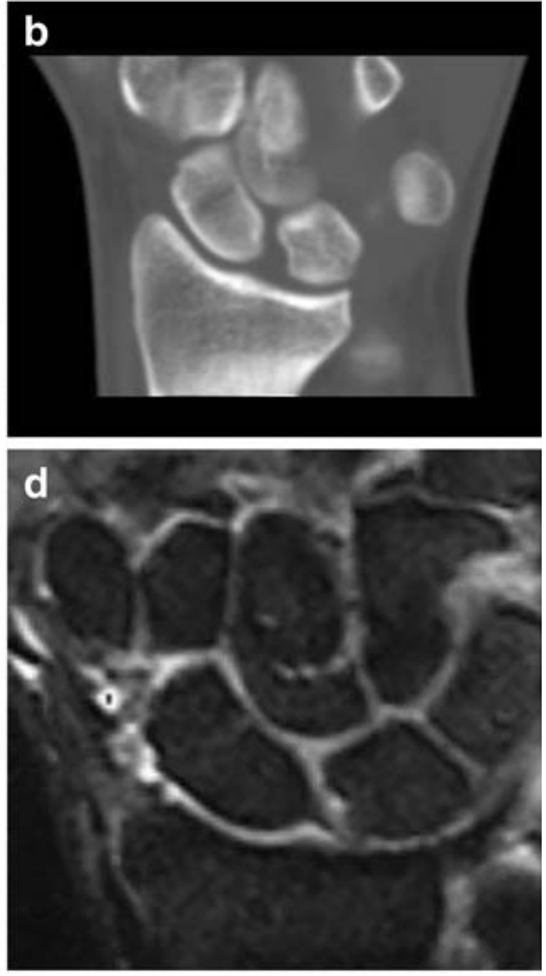

splint removed after a week and was then instructed to stay away from practice during 6 weeks but was allowed to use her hand in everyday activities. Two months after the initial injury, she had another CT scan taken which demonstrated partial union (Fig. 5b). She went back in training shortly thereafter and has resumed her professional career without further symptoms from the injured wrist.

\section{Conclusions}

This report demonstrates that union can occur in undisplaced and minimally displaced scaphoid waist fractures without or with significantly shorter time of immobilization than generally recommended. In the particular cases presented, there may have been several factors that contributed to the
Fig. 5 a MRI of the left wrist a few hours after a fall demonstrating a scaphoid waist fracture and bone oedema, $\mathbf{b}$ signs of fracture union at a CT scan 3 months after the injury
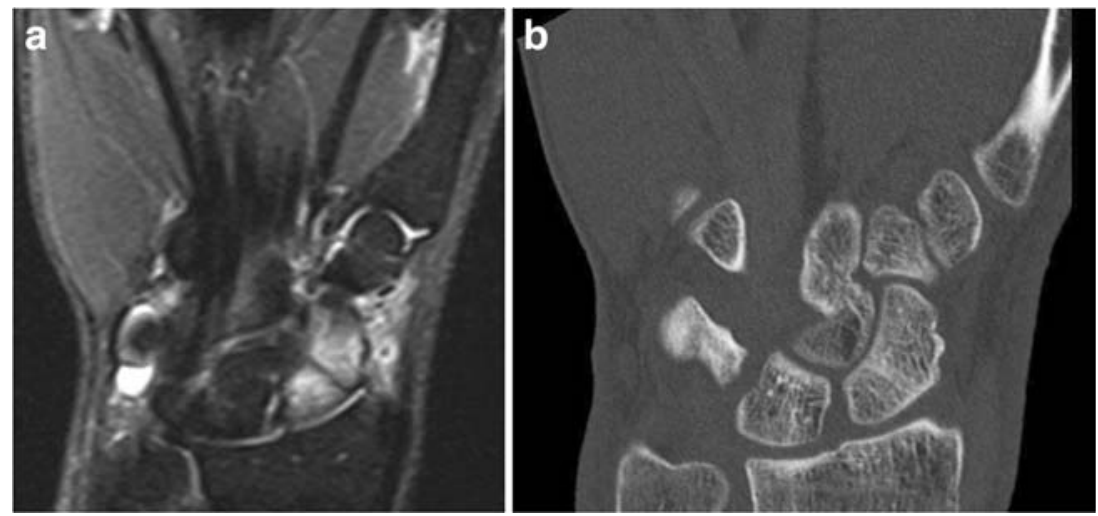
uneventful union. All patients were young and healthy, and the fractures were predominantly transverse and presumably relatively stable. The fact that there was no or minimal displacement of the fractures indicates a less likelihood of severe injury to the surrounding soft tissues. There were no radiographic signs of circulatory disturbances in any of the examinations. Some of the fractures may even have been incomplete in accordance with the classification by Herbert [6]. This classification was based on conventional radiographs, while today CT scans and MRI are often advocated to diagnose fractures not visible on plain x-rays. The use of these modalities may however infer a risk of overtreatment since the presented cases indicate that undisplaced fractures could be inherently stable and heal without treatment if protected from additional trauma or heavy loading.

Despite, based on only the five cases presented here, our observations indicate that some of the patients with undisplaced scaphoid waist fractures are at risk of immobilization for unnecessarily long periods if traditional recommendations are followed. If some fractures will unite without or with shorter immobilization than has been generally perceived, the argument for acute internal fixation in these cases will also be significantly weakened. It appears that inherent fracture stability is of utmost importance for the healing potential, and to guide treatment, perhaps more emphasis should aim at identifying factors indicating fracture instability such as vertical or oblique fracture patterns. On the other hand, it has been demonstrated that not all fractures appearing as undisplaced at conventional radiographs are stable $[1,2]$ and future use of dynamic radiographic examinations may prove of value. Future studies on the influence on fracture healing by inherent fracture stability, fracture pattern and vascularity would be of value to ascertain the optimal treatment of scaphoid waist fractures.

Acknowledgements Open access funding provided by Linköping University.

Author Contribution LA and YT have been involved in gathering the cases, and all three authors have been involved in writing the manuscript.

\section{Compliance with Ethical Standards}

Conflict of Interest The authors declare that they have no conflict of interests.
Ethical Approved This case report was reviewed and approved by the Internal Review Board of the University Hospital in Linköping Sweden. They determined that the case report did not need to be further reviewed by the South east regional ethical board of Sweden.

Informed Consent All patients in this case report provided written informed consent authorizing publication of the included information and radiographs.

Open Access This article is licensed under a Creative Commons Attribution 4.0 International License, which permits use, sharing, adaptation, distribution and reproduction in any medium or format, as long as you give appropriate credit to the original author(s) and the source, provide a link to the Creative Commons licence, and indicate if changes were made. The images or other third party material in this article are included in the article's Creative Commons licence, unless indicated otherwise in a credit line to the material. If material is not included in the article's Creative Commons licence and your intended use is not permitted by statutory regulation or exceeds the permitted use, you will need to obtain permission directly from the copyright holder. To view a copy of this licence, visit http://creativecommons.org/licenses/by/4.0/.

\section{References}

1. Adolfsson L, Lindau T, Arner M. Acutrak screw fixation versus cast immobilisation for undisplaced scaphoid waist fractures. J Hand Surg Br. 2001;26:192-5.

2. Buijze GA, Jorgsholm P, Thomsen NO, Bjorkman A, Besjakov J, Ring D. Factors associated with arthroscopically determined scaphoid fracture displacement and instability. J Hand Surg Am. 2012;37: 1405-10.

3. Dias JJ, Wildin CJ, Bhowal B, Thompson JR. Should acute scaphoid fractures be fixed? A randomized controlled trial. J Bone Joint Surg Am. 2005;87:2160-8.

4. Grewal R, King G. Percutaneous screw fixation led to faster recovery and return to work than immobilization for fractures of the waist of the scaphoid. J Bone Joint Surg Am. 2008;90:1793.

5. Haddad FS, Goddard NJ. Acute percutaneous scaphoid fixation. A pilot study J Bone Joint Surg Br. 1998;80:95-9.

6. Herbert TJ, Fisher WE. Management of the fractured scaphoid using a new bone screw. J Bone Joint Surg Br. 1984;66:114-23.

7. Slade JF 3rd, Gillon T. Retrospective review of 234 scaphoid fractures and nonunions treated with arthroscopy for union and complications. Scand J Surg. 2008;97:280-9.

Publisher's Note Springer Nature remains neutral with regard to jurisdictional claims in published maps and institutional affiliations. 